\section{NP-018 BUILDING CAPACITY WITHIN A HEPATITIS C TREATMENT MODEL. THE VALIDATION PROCESS FOR A HEPATITIS C PRE-TREATMENT PHARMACIST ASSESSMENT COMPLEX INTERVENTION TOOLKIT}

${ }^{1} \mathrm{M}$ Coghlan, ${ }^{2} \mathrm{~A} O \mathrm{O}^{\prime}$ Leary, ${ }^{3} \mathrm{G}$ Melanophy, ${ }^{4} \mathrm{~S}$ Norris, ${ }^{5} \mathrm{C}$ Bergin. ${ }^{1}$ Pharmacy Department, St. James's Hospital Dublin, School of Medicine, Trinity College Dublin; ${ }^{2}$ National Centre for Pharmacoeconomics, St. James's Hospital, Dublin 8; 'Pharmacy Department, St. James's Hospital, Dublin; ${ }^{4}$ Department of Hepatology, St. James's Hospital, Dublin; ${ }^{5}$ Department of GU Medicine and Infectious Diseases, St. James's Hospital, Dublin

\subsection{6/ejhpharm-2021-eahpconf.365}

Background and importance The pre-treatment pharmacist assessment is a complex intervention toolkit (CIT) which has been designed to support devolvement of Hepatitis C (HCV) treatment to primary care providers including pharmacists. It combines all aspects of pre-treatment assessment into a proforma to ensure optimum $\mathrm{HCV}$ treatment selection.

Aim and objectives To assess the validity of the PTPA via a matched cohort study.

Material and methods Pharmacists were invited to participate in this study to review HCV case vignettes. Participants were divided into two groups using a concealed randomisation method (Group A = CIT use; Group B = case review as per current standard practice). A random sample of anonymised cases were selected from the Irish HCV treatment registry using selected co-variates (eg, fibrosis stage). A sample size of 56 cases per group was calculated. The primary endpoint was selection of the optimum treatment regimen. Secondary endpoints included time to completion, detection of drug-drug interactions (DDIs) and patient interventions. Statistical analysis was completed to assess variation between groups.

Results A total of 56 cases were completed per group. CIT use was associated with selection of optimum HCV treatment in $92.9 \%$ of cases, compared with $60.7 \%$ of cases in group B $(\mathrm{p}<0.05)$. DDI detection rates increased with CIT use $(74.8 \%$ vs $47.1 \% ; \mathrm{p}<0.05)$. CIT users proposed an average of 3.5 interventions per case versus 2 interventions per case in Group B. The CIT was associated with a longer median completion time (20 versus 15 minutes, however this difference was not statistically significant (p 0.06).

Conclusion and relevance The findings of this study confirm the effectiveness of the CIT. The potential for pharmacists working in all practice environments in Ireland to make a robust contribution to HCV treatment, can be supported using this CIT. This type of capacity building is key to upscaling the model of care to achieve elimination targets.

\section{REFERENCES AND/OR ACKNOWLEDGEMENTS}

1. Zhou K, Fitzpatrick T, Walsh N, Kim JY, Chou R, Lackey M, et al. Interventions to optimise the care continuum for chronic viral hepatitis: a systematic review and meta-analyses. The Lancet Infectious diseases 2016.

2. Meyer JP, Moghimi Y, Marcus R, Lim JK, Litwin AH, Altice FL. Review: Evidencebased interventions to enhance assessment, treatment, and adherence in the chronic Hepatitis C care continuum. International Journal of Drug Policy 2015;26:922-35.

3. Craig P, Dieppe P, Macintyre S, Michie S, Nazareth I, Petticrew M. Developing and evaluating complex interventions: the new Medical Research Council guidance. BMJ (Clinical research ed). 2008;337: a1655.

4. Campbell NC, Murray E, Darbyshire J, Emery J, Farmer A, Griffiths F, et al. Designing and evaluating complex interventions to improve health care. BMJ (Clinical research ed). 2007;334(7591):455-9.

\section{NP-019 DEVELOPMENT OF A CONVERSION FACTOR BETWEEN DEFINED DAILY DOSES OF ADULTS AND NEONATES}

C Villanueva Bueno*, ${ }^{1} \mathrm{MV}$ Gil Navarro, ${ }^{1} \mathrm{E}$ Montecatine Alonso, ${ }^{2} \mathrm{~F}$ Jiménez Parrilla, ${ }^{3} \mathrm{MJ}$ Cabañas Poy, ${ }^{4} \mathrm{M}$ González López, ${ }^{5} \mathrm{C}$ Martínez Fernández Llamazares, ${ }^{6} \mathrm{OW}$ Neth. 'Department of Pharmacy, Institute of Biomedicine of Seville (IBIS), Hospital Universitario Virgen del Rocío, Sevilla, Spain; ${ }^{2}$ Department of Neonatology Hospital Universitario Virgen del Rocio, Sevilla, Spain; ${ }^{3}$ Department of Pharmacy, Hospital Universitario Vall d'Hebron, Barcelona, Spain; ${ }^{4}$ Department of Neonatology, Hospital Materno-Infantil Regional de Málaga, Málaga, Spain; ${ }^{5}$ Department of Pharmacy, Hospital Gregorio Marañón. Instituto de Investigación Sanitaria Gregorio Marañón.Madrid, Spain; ${ }^{6}$ Paediatric Infectious Diseases, Rheumatology and Immunology Unit, Hospital Universitario Virgen del Rocío, Sevilla, Institute of Biomedicine of Seville (IBIS), Spain

\subsection{6/ejhpharm-2021-eahpconf.366}

Background and importance Absence of a standardised method to measure antimicrobial consumption in the neonatal population

Aim and objectives Establish a conversion factor that makes possible to relate the defined daily dose (DDD) of the adult population and the DDD of the neonatal population.

Materials and methods National, multicentre and retrospective study. It had the participation of 10 third-second level hospitals and a multidisciplinary team: hospital pharmacists, paediatricians, neonatologists and infectious disease experts.

First, after selecting the antimicrobials for study based on the literature and routine clinical practice, the Delphi methodology was used to agree on the recommended dose for its most common indication in the neonatal population. Two rounds of consultation with the group of experts were carried out. For those antimicrobials whose dose was not agreed upon, the dose established in Pediamecum was selected.

The weight and gestational age of the neonates ( $<1$ month) admitted to 6 hospitals during 2018 were collected through the admission records or own records of the pharmacy or neonatology services of these hospitals. The mean, median, standard deviation and maximum and minimum of each of the variables were calculated.

Subsequently, the DDD in grams of each antimicrobial were calculated.

Finally, the conversion factor was obtained by comparing the DDD designed for neonates (DDDn) with the DDD validated by the WHO for adults (DDDa).

For the analysis of the results, the statistical package IBM SPSS Statistics version 19 was used.

Results 4820 neonates were selected. The mean weight was $2687 \mathrm{~g}(6080-440)$.

The conversion factor (DDDn/DDDa) of the parenterally administered antimicrobials were: amikacin (0.04), amoxicillin (0.03), amoxicillin-clavulanate (0.09), ampicillin (0.04), liposomal amphotericin B (0.38), azithromycin (0.05), aztreonam (0.06), cefazolin (0.04), cefepime (0.07), cefotaxime $(0.07)$, ceftazidime (0.07), ceftriaxone (0.07), ciprofloxacin(0.05), erythromycin(0.13), fluconazole(0.08), gentamicin (0.04), imipenem-cilastatin (0.07), linezolid (0.07), meropenem (0.04), metronidazole (0.03), micafungin $(0.05)$, penicillin $G$ sodium (0.03), piperacillin-tazobactam (0.04), teicoplanin (0.04), and vancomycin (0.04)

Those obtained from oral antimicrobials were: amoxicillin (0.05), amoxicillin-clavulanate (0.08), azithromycin (0.09), cefadrozil (0.04), cefixime (0.05), ciprofloxacin (0.05), clindamycin 